\title{
Article \\ A Convenient Approach towards the Synthesis of ADMDP Type Iminosugars and Nojirimycin Derivatives from Sugar-Derived Lactams
}

\author{
Piotr Szcześniak DD, Barbara Grzeszczyk and Bartłomiej Furman * \\ Institute of Organic Chemistry, Polish Academy of Sciences, Kasprzaka 44/52, 01-224 Warsaw, Poland; \\ pszczesniak@icho.edu.pl (P.S.); bgrzeszczyk@icho.edu.pl (B.G.) \\ * Correspondence: bartlomiej.furman@icho.edu.pl
}

check for

updates

Citation: Szcześniak, P.; Grzeszczyk,

B.; Furman, B. A Convenient

Approach towards the Synthesis of ADMDP Type Iminosugars and Nojirimycin Derivatives from Sugar-Derived Lactams. Molecules 2021, 26, 5459. https://doi.org/ $10.3390 /$ molecules 26185459

Academic Editor: Robert J. Linhardt

Received: 28 July 2021

Accepted: 2 September 2021

Published: 8 September 2021

Publisher's Note: MDPI stays neutral with regard to jurisdictional claims in published maps and institutional affiliations.

Copyright: (c) 2021 by the authors. Licensee MDPI, Basel, Switzerland. This article is an open access article distributed under the terms and conditions of the Creative Commons Attribution (CC BY) license (https:// creativecommons.org/licenses/by/ $4.0 /)$.
Abstract: An efficient method for the synthesis of nojirimycin- and pyrrolidine-based iminosugar derivatives has been developed. The strategy is based on the partial reduction in sugar-derived lactams by Schwartz's reagent and tandem stereoselective nucleophilic addition of cyanide or a silyl enol ether dictated by Woerpel's or diffusion control models, which affords amino-modified iminosugars, such as ADMDP or higher nojirimycin derivatives.

Keywords: sugar-derived lactams; sugar-derived imines; iminosugars; ADMDP; nojirimycin derivatives

\section{Introduction}

Iminosugars are a large group of carbohydrate analogues that have received a lot of attention due to their ability to inhibit enzymes responsible for the formation or cleavage of glycosidic bonds [1-5]. They have been recognized as potent active agents in the treatment of various diseases, such as diabetes, lysosomal storage disorder, viral infection, and cancer [1,6-9]. Some drugs based on iminosugars are already in use, while a few others are in clinical trials. For example, Glyset ${ }^{\circledR}$ is used to treat non-insulin-dependent diabetes, and Zavesca ${ }^{\circledR}$ is used in Gaucher's disease treatment. Another piperidine-originated iminosugar, 1-deoxynojirimycin (DNJ), and its amino derivative (4b), displayed similar inhibition to $\alpha$-glucosidase [10]. $\alpha$-Homonojirimycin is a powerful $\alpha$-glucosidase inhibitor and is expected to be a drug candidate for antidiabetic therapy [11].

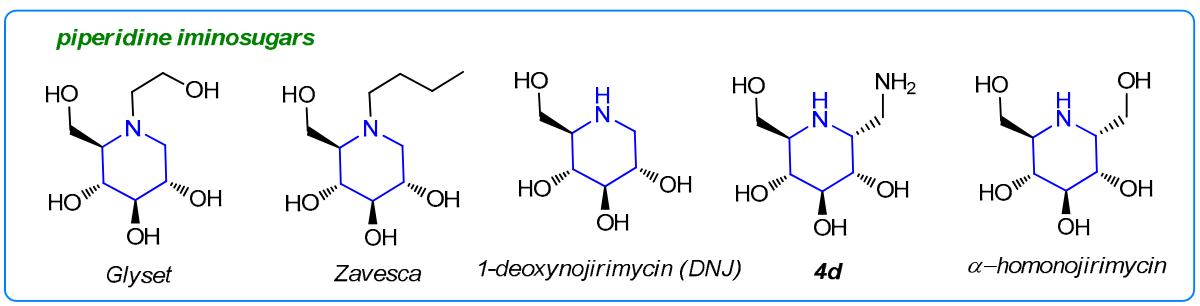

Pyrrolidine-derived iminosugars are also promising drug candidates [1]. For example, 2,5-dihydroxymethyl-3,4-di-hydroxypyrrolidine (DMDP) is a known glycosidase inhibitor. In turn, 1-aminodeoxy-DMDP (ADMDP), an unnatural product that possesses inhibitory activity against $n$-acetyl- $\beta$-glucosamidase [12], as well as other DMDP analogues [13-16], have been found to be potential drug candidates for osteoarthritis [13-16] and bacterial infections $[16,17]$. 


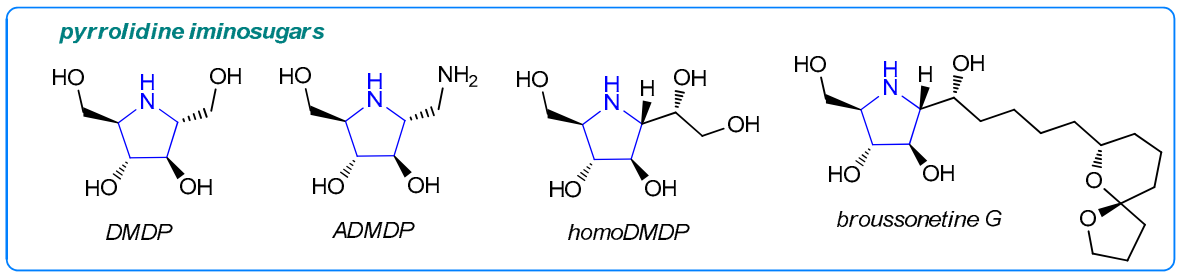

The high potential of amino-iminosugars has resulted in the development of various methods for their synthesis. The first preparation methods of ADMDP and its isomers were concerned with the transformation of naturally occurring DMDP (and its stereo analogues) by selective conversion of one of the side-chain hydroxyl groups into an amino functionality [13].Other strategies include intramolecular cyclization of C2-symmetric amino alcohols [18], transformation of non-racemic $\gamma$-lactams [19], nucleophilic ring opening of sugar-derived bis-aziridines [20], and nucleophilic addition of cyanide to sugar-derived cyclic nitrones, followed by reduction [21,22]. Stocker and colleagues [23] reported the synthesis of ADMDP and analogues by carbamate annulation, whereas the group of Ramesh reported their synthesis via deamination of glycals [24]. In our previous work, we found that the furanosyl and pyranosyl imines can be obtained from sugar-derived lactams via a Schwartz's-reagent-mediated partial reduction in amide function [25]. Moreover, we demonstrated that these cyclic imines could be directly used (without isolation) in further transformations [25-27], leading to, for example, highly functionalized pyrrolidine and piperidine iminosugars via a tandem one-pot reduction/Grignard reagent or allyltributyltin addition sequence (Scheme 1a) [25]. This developed protocol was applied for the preparation of two pyrrolidine derivatives: 6-deoxy-DMDP and radicamine B [25]. Developing our group's program on reductive sugar-derived lactam functionalization [25-27], we report herein the flexible and robust access to amino-iminosugars, such as ADMDP and higher derivatives, via a gluco- or arabino-lactam reduction/cyanide ion or silyl enol ether addition sequence (Scheme $1 b$ ).

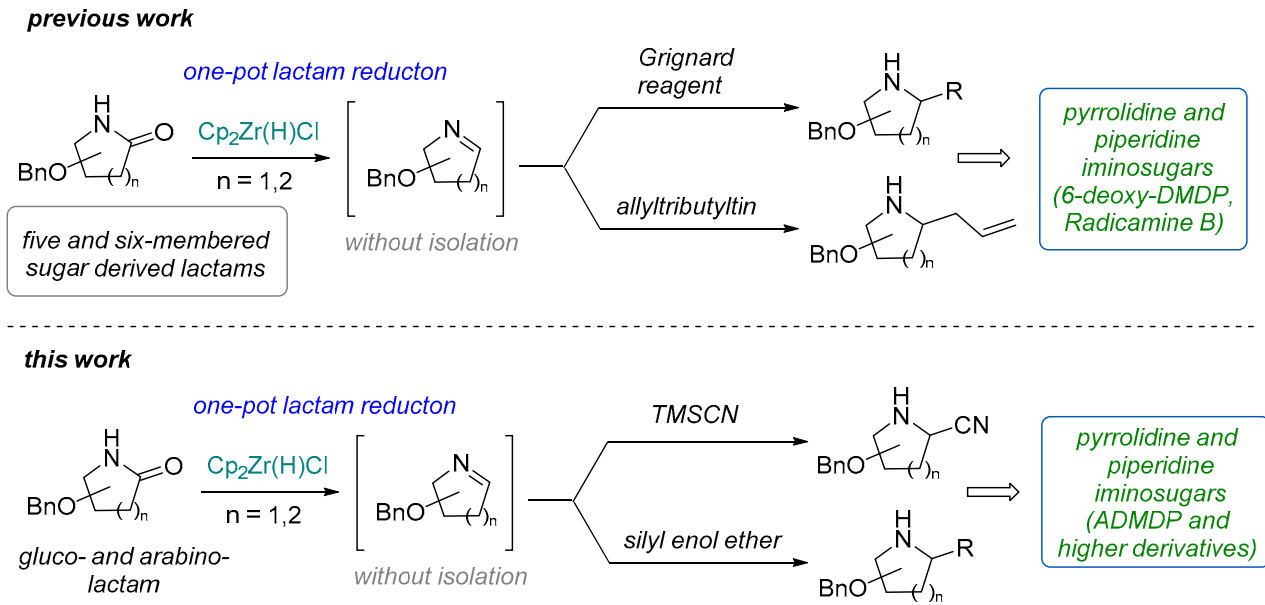

Scheme 1. The previous work (a) and this work (b).

\section{Results and Discussion}

Our synthesis of amino-modified iminosugars started with the synthesis of initial sugar-derived lactams. We chose gluco-1a and arabino-1 $\mathbf{b}$ lactam, obtained from the corresponding commercially available glucose and arabinose, as model starting materials for further studies (Figure 1) [25,28]. 

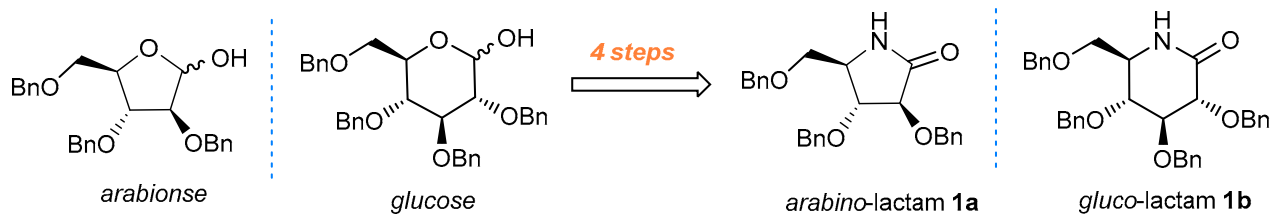

Figure 1. General route to sugar-derived lactams (1a) and (1b).

With appropriate substrates (1a and $\mathbf{1 b})$ in hand, we investigated a one-pot lactam reduction/cyanide addition reaction (Scheme 2). Arabino-lactam 1a was treated with 1.6 equiv of Schwartz's reagent $\mathrm{Cp}_{2} \mathrm{Zr}(\mathrm{H}) \mathrm{Cl}$, leading to appropriate imine 2a in almost quantitative yield according to the NMR analysis of the crude reaction mixture. The progress of the reaction can easily be followed thanks to simple direct observation: initially white, then heterogeneous mixture turns to a clear solution upon reduction completion (approximately $10 \mathrm{~min}$ ). Subsequent treatment of the in situ generated imine 2a with trimethylsilyl cyanide (2.0 equiv) in the presence of TMSOTf (1.0 equiv) gave a mixture of two nitriles 3a and 2-epi-3a in a ratio of $60: 40$ and with an overall yield of $88 \%$ after two steps (Scheme 2). Hydrogenation of 3a and 2-epi-3a under the conditions reported by Cheng et al. [22] gave ADMDP and its 2-epimer (2-epi-ADMDP) in 73\% and 69\% yield, respectively (Scheme 2). The same approach was successfully applied for the synthesis of the six-membered amino-iminosugar. Thus, treatment of gluco-lactam $1 \mathbf{b}$ with Schwartz's reagent, followed by TMSOTf-mediated addition of trimethylsilyl cyanide gave nitrile $3 \mathbf{b}$ in $65 \%$ yield as a single isomer. Its hydrogenolysis under the conditions presented above afforded gluconojirimycin derivative $4 \mathrm{~b}$ in $66 \%$ yield (Scheme 2). Elsewhere, Cheng et al. [22] reported the transformation of nitrile 2-epi-3a to 2-epi-DMDP. We expect that the same strategy can be used for the synthesis of DMDP and $\alpha$-homonojirimycin starting form $3 \mathbf{a}$ and $\mathbf{3 b}$, respectively (Scheme 2).



Scheme 2. Reagents and conditions: (a) $\mathrm{Cp}_{2} \mathrm{Zr}(\mathrm{H}) \mathrm{Cl}$ (1.6 equiv), THF, RT $10 \mathrm{~min}$; (b) TMSOTf (1.0 equiv), TMSCN (2.0 equiv), THF, $-25{ }^{\circ} \mathrm{C}$ to RT, overall yield for two steps $3 \mathrm{a}$ 88\% d.r. 60:40, 3b 65\% d.r. > 95:5; (c) $\mathrm{H}_{2}, \mathrm{Pd}(\mathrm{OH})_{2}, \mathrm{MeOH}, \mathrm{AcOH}, \mathrm{RT}$. ADMDP 73\%, 2-epi-ADMDP 69\%, 4b 66\%.

Configuration of the newly generated stereochemical center in nitriles 3a, 2-epi-3a, and $3 \mathbf{b}$ have been established by NOE experiments. Addition of TMSCN to gluco-imine $2 \mathbf{b}$ proceeded syn to the $\mathrm{BnO}$ substituent at the $\mathrm{C}-3$ position. This is a result of conformational control of the process, according to Woerpel's model (Figure 2a) [29-34]. In the latter case, the nucleophilic addition of TMSCN to arabino-imine $\mathbf{2 a}$ occurs with low stereoselectivity, leading to the separable mixture of isomers $3 \mathbf{a}$ and 2-epi-3a in a ratio of 60:40.

The same modest diastereoselectivity was observed by Woerpel in the reaction of TMSCN with cyclic oxocarbenium ions [35]. Woerpel postulated that this effect can be attributed to the high reactivity of the nucleophilic species involved [36]. The silyl cyanides require activation by a nucleophile in solution (such as the counterion of the Lewis acid) 
to form a pentacoordinate siliconate ion that transfers the cyano group. This activation step is slow relative to nucleophilic addition. A detailed, step-by-step description of this process has been explained by Woerpel in his vital paper from 2006 [35]. It can be assumed that a similar effect applies in the TMSOTf-mediated addition of TMSCN to arabino-imine 2a (Figure 2b).

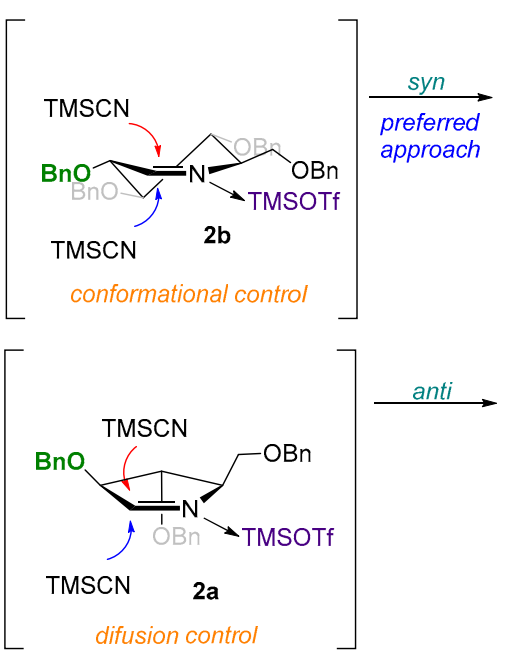

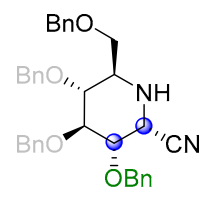

3b

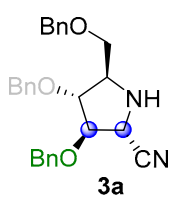

(b)

Figure 2. Stereocontrol of addition of TMSCN to gluco $\mathbf{2 b -}$ - (a) and arabino $\mathbf{2 a -}$ - (b) derived cyclic imines.

Subsequently, other types of nucleophiles, such as silyl enol ethers $\mathbf{5 a}, \mathbf{5 b}$, and $\mathbf{5 c}$, were examined in the tandem one-pot reduction/nucleophilic addition protocol (Scheme 3). Gluco-lactam $\mathbf{1 b}$ was selected as a model compound for this part of the study. On the basis of previous examples, a solution of gluco-lactam $\mathbf{1 b}$ was added to a suspension of Schwartz reagent (1.6 equiv) in THF. After the initially white suspension turned clear, the enol silyl ether (2 equiv) and $\mathrm{Yb}(\mathrm{OTf})_{3}(10 \mathrm{~mol} \%)$ were added at $-25^{\circ} \mathrm{C}$, and the reaction mixture was warmed slowly to room temperature. The desired functionalized piperidines $\mathbf{6 a}, \mathbf{6} \mathbf{b}$, and $6 \mathrm{c}$ were obtained in $88 \%, 68 \%$, and $43 \%$ yields, respectively, and with a high level of stereoselectivity (Scheme 3). The stereochemical structure of piperidines $\mathbf{6 a}, \mathbf{6} \mathbf{b}$, and $\mathbf{6 c}$ were confirmed by NOE experiments. The stereochemistry between the substituent at C-2 and the benzyloxy group at C-3 was determined to be in a syn arrangement for all piperidines $6 \mathbf{a}$, $\mathbf{6 b}$, and $\mathbf{6 c}$. We are dealing here with the same model of stereocontrol as shown in Figure 2a. Obtained compounds $\mathbf{6 a}-\mathbf{c}$ are attractive building blocks for the syn thesis of functionalized nojirimycin derivatives.

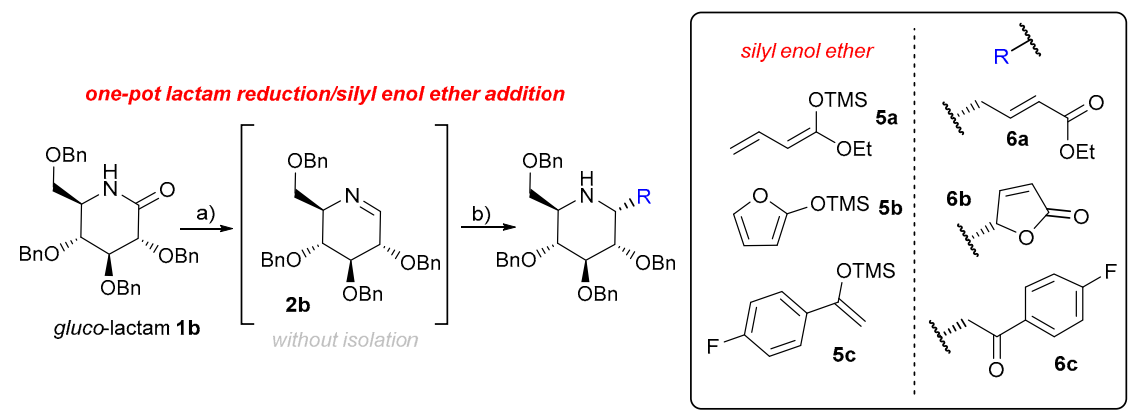

Scheme 3. Reagents and conditions: (a) $\mathrm{Cp}_{2} \mathrm{Zr}(\mathrm{H}) \mathrm{Cl}$ (1.6 equiv), THF, RT $10 \mathrm{~min}$; (b) $\mathrm{Yb}(\mathrm{OTf})_{3}$ ( 0.1 equiv) silyl enol ether $5 \mathbf{a}, 5 \mathbf{b}$, or $5 \mathbf{c}\left(2.0\right.$ equiv.), THF, $-25^{\circ} \mathrm{C}$ to RT, overall yield for two steps 6a $88 \%$ d.r. $>95: 5, \mathbf{6 b} 68 \%$ d.r. 75:22; $6 \mathbf{c} 43 \%$ d.r. $>95: 5$.

\section{Conclusions}

In conclusion, we have presented a convenient route for the synthesis of aminomodified iminosugars, such as ADMDP, based on the reductive activation of sugar-derived 
lactones and silyl cyanides. The presented method is one of the shortest preparations of ADMDP. In contrast to similar strategies based on nucleophilic addition to cyclic nitrones, our method uses the more easily available and stable lactams. An additional advantage of the current method over nitrone-based strategies [21,22] is the fact that it can also be successfully applied to the synthesis of six-membered analogues of ADMDP (4d). The former method is limited due to the more challenging synthesis and limited stability of six-membered nitrones compared to their five-membered counterparts [37]. Moreover, we have presented that other types of nucleophile, such as silyl enol ethers, can be applied, leading to functionalized nojirimycin derivatives.

\section{Experimental Section}

\subsection{General Information}

${ }^{1} \mathrm{H}$ NMR and ${ }^{13} \mathrm{C}$ NMR spectra were recorded on Varian VNMRS 500 and Varian VNMRS 600 spectrometers, in $\mathrm{CDCl}_{3}$, unless otherwise stated, and with TMS used as an internal standard. Chemical shifts $(\delta)$ were given in ppm and coupling constants $(J)$ were given in Hertz $(\mathrm{Hz})$. Infrared spectra were recorded on an FT-IR Jasco 6200 and FT-IR Spectrum 2000 Perkin Elmer spectrophotometer. High-resolution mass spectra were recorded on an ESI-TOF Mariner Spectrometer, SYNAPT G2-S HDMS, or AMD 604 mass spectrometer. Optical rotations were measured with a Jasco P-2000 polarimeter. Thin-layer chromatography was performed on Merck aluminium sheet Silica Gel 60 F254. Column chromatography was carried out using Merck silica gel (230-400 mesh).

\subsection{One-Pot Reduction/TMSCN Addition to Sugar-Lactams $\mathbf{1} a$ and $\mathbf{1} b$-General Procedure for} The Synthesis of $3 a, 2-E p i-3 a$, and $3 b$

A solution of sugar lactam $\mathbf{1 a}$ or $\mathbf{1 b}(0.5 \mathrm{mmol})$ in THF $(5 \mathrm{~mL})$ was added dropwise to a suspension of $\mathrm{Cp}_{2} \mathrm{Zr}(\mathrm{H}) \mathrm{Cl}$ (1.6 equiv, $0.8 \mathrm{mmol}, 206 \mathrm{mg}$ ) in THF (5 mL) under an argon atmosphere. The mixture was stirred until the white suspension disappeared (ca. $10 \mathrm{~min}$ ) to form a clear solution. Next, the imine solution was cooled to $-25^{\circ} \mathrm{C}$ and TMSOTf was added ( 1.0 equiv, $0.5 \mathrm{mmol}, 60 \mu \mathrm{L}$ ). The mixture was stirred for $10 \mathrm{~min}$ and TMSCN (2.0 equiv, $1.0 \mathrm{mmol}, 125 \mu \mathrm{L}$ ) was added dropwise. The mixture was warmed to room temperature and stirred overnight. The reaction was quenched by addition of aq. $\mathrm{NaHCO}_{3}$ and stirred for $30 \mathrm{~min}$. After dilution with $\mathrm{Et}_{2} \mathrm{O}(5 \mathrm{~mL})$, the aqueous phase was separated and washed twice with $\mathrm{Et}_{2} \mathrm{O}(2 \times 5 \mathrm{~mL})$. The combined organic solutions were dried over anhydrous $\mathrm{MgSO}_{4}$, filtered, and solvents were removed under reduced pressure. The residue was chromatographed on silica gel to afford the corresponding amine derivatives 3a, 2-epi-3a, and $3 b$.

(2R,3R,4R,5R)-3,4-Bis(benzyloxy)-5-(benzyloxymethyl)pyrrolidine-2-carbonitrile (3a): (major isomer), colorless oil; 88\% (overall, isolated yield for two steps, both isomers); $d r$ 60:40 (determined by ${ }^{1} \mathrm{H}$ NMR of crude reaction mixture); $\mathrm{R}_{f} 0.34$ (1:2 AcOEt/hexanes); chromatography (1:3 AcOEt/hexanes); $[\alpha]_{\mathrm{D}}+23.6\left(c 0.2 \mathrm{CH}_{2} \mathrm{Cl}_{2}\right) ;{ }^{1} \mathrm{H}$ NMR $\left(600 \mathrm{MHz}, \mathrm{CDCl}_{3}\right)$ ঠ: 7.38-7.20 (m, 15H), $4.64(\mathrm{~d}, J 11.9 \mathrm{~Hz}, 1 \mathrm{H}), 4.59(\mathrm{~d}, J 11.9 \mathrm{~Hz}, 1 \mathrm{H}), 4.53(\mathrm{~d}, J 11.9 \mathrm{~Hz}, 1 \mathrm{H})$, $4.49(\mathrm{~d}, J 11.9 \mathrm{~Hz}, 1 \mathrm{H}), 4.48-4.47(\mathrm{~m}, 2 \mathrm{H}), 4.14-4.09(\mathrm{~m}, 2 \mathrm{H}), 3.93-3.89(\mathrm{~m}, 1 \mathrm{H}), 3.60-3.53(\mathrm{~m}$, 2H), 3.38-3.33 (m, 1H); ${ }^{13} \mathrm{C}$ NMR (151 MHz, $\left.\mathrm{CDCl}_{3}\right)$ 8: 137.8, 137.4, 136.9 128.6, 128.5, 128.4, 128.1, 128.0, 127.95, 127.74, 127.72, 117.6, 83.3, 82.8, 73.3, 72.5, 72.0, 70.4, 62.7, 51.2; IR (film) v: 3354, 3030, 2924, 2854, 2246, 1495, 1454, 1376, 1364, 1206, 1091, 1072, 733, $695 \mathrm{~cm}^{-1}$; HRMS (ESI-TOF) $m / z$ calculated for $\mathrm{C}_{27} \mathrm{H}_{29} \mathrm{~N}_{2} \mathrm{O}_{3}\left[\mathrm{M}+\mathrm{H}^{+}\right] 429.2178$, found 429.2184 .

(2S,3R,4R,5R)-3,4-Bis(benzyloxy)-5-(benzyloxymethyl)pyrrolidine-2-carbonitrile (2-epi-3a): (minor isomer), colorless oil; $\mathrm{R}_{f} 0.45$ (1:2 AcOEt/hexanes); chromatography (1:3 AcOEt/hexanes); $[\alpha]_{\mathrm{D}}-4.7\left(c 0.85, \mathrm{CH}_{2} \mathrm{Cl}_{2}\right) ;{ }^{1} \mathrm{H} \mathrm{NMR}\left(600 \mathrm{MHz}, \mathrm{CDCl}_{3}\right)$ 8: 7.39-7.24 $(\mathrm{m}, 14 \mathrm{H}), 4.59(\mathrm{~d}, J 11.7 \mathrm{~Hz}, 1 \mathrm{H}), 4.55(\mathrm{~d}, J 11.9 \mathrm{~Hz}, 1 \mathrm{H}), 4.52(\mathrm{~d}, J 9.5 \mathrm{~Hz}, 1 \mathrm{H}), 4.51-4.45$ $(\mathrm{m}, 3 \mathrm{H}), 4.3-4.27(\mathrm{~m}, 1 \mathrm{H}), 4.06-4.03(\mathrm{~m}, 1 \mathrm{H}), 3.87-3.83(\mathrm{~m}, 1 \mathrm{H}), 3.62-3.58(\mathrm{~m}, 1 \mathrm{H}), 3.52-3.46$ $(\mathrm{m}, 2 \mathrm{H}) ;{ }^{13} \mathrm{C}$ NMR $\left(151 \mathrm{MHz}, \mathrm{CDCl}_{3}\right) \delta: 137.7,137.5,136.6,128.6,128.4,128.3,127.99,128.0$, $127.81,127.78,127.7,119.0,87.2,84.0,73.3,72.6,72.2,69.5,62.1,51.7$; IR (film) v: 3343, 3064, 
3031, 2922, 2863, 2240, 1496, 1454, 1363, 1097, 1028, 738, $698 \mathrm{~cm}^{-1}$; HRMS (ESI-TOF) $\mathrm{m} / \mathrm{z}$ calculated for $\mathrm{C}_{27} \mathrm{H}_{29} \mathrm{~N}_{2} \mathrm{O}_{3}\left[\mathrm{M}+\mathrm{H}^{+}\right] 429.2178$, found 429.2189 .

(2S,3S,4R,5R,6R)-3,4,5-tris(benzyloxy)-6-(benzyloxymethyl)piperidine-2-carbonitrile (3b): white crystals, $\mathrm{mp} 144^{\circ} \mathrm{C}$; $65 \%$ (overall, isolated yield for two steps, both isomers); $d r>95: 5$ (from 1H NMR of the crude reaction mixture); $\mathrm{R}_{f} 0.32$ (1:5 AcOEt/hexanes); chromatography $\left(1: 7 \mathrm{AcOEt} /\right.$ hexanes); $[\alpha]_{\mathrm{D}}+52.6\left(c, 0.78 \mathrm{CH}_{2} \mathrm{Cl}_{2}\right) ;{ }^{1} \mathrm{H} \mathrm{NMR}\left(600 \mathrm{MHz}, \mathrm{CDCl}_{3}\right)$

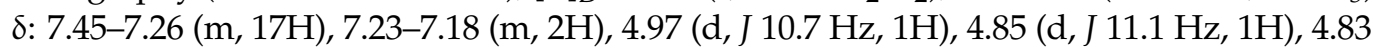
$(\mathrm{d}, J 10.8 \mathrm{~Hz}, 1 \mathrm{H}), 4.77(\mathrm{~d}, J 11.9 \mathrm{~Hz}, 1 \mathrm{H}), 4.68(\mathrm{~d}, J 11.9 \mathrm{~Hz}, 1 \mathrm{H}), 4.51-4.46(\mathrm{~m}, 2 \mathrm{H}), 4.41$ (d, J $11.9 \mathrm{~Hz}, 1 \mathrm{H}), 4.08(\mathrm{~d}, J 5.6 \mathrm{~Hz}, 1 \mathrm{H}), 3.87-3.81(\mathrm{~m}, 1 \mathrm{H}), 3.69$ (dd, J 9.4, $2.4 \mathrm{~Hz}, 1 \mathrm{H}), 3.59$ (dd, J 9.4, 5.6 Hz, 1H), $3.36(\mathrm{dd}, J$ 9.2, $7.3 \mathrm{~Hz}, 1 \mathrm{H}), 3.27-3.19(\mathrm{~m}, 2 \mathrm{H}) ;{ }^{13} \mathrm{C}$ NMR $(151 \mathrm{MHz}$, $\mathrm{CDCl}_{3}$ ) $\delta: 138.4,138.0,137.6,137.5,128.6,128.5,128.41,128.40,128.1,128.0,127.97,127.90$, $127.87,127.77,127.7,117.5,84.2,79.0,78.6,76.0,75.1,73.3,73.2,69.9,55.6,49.7 ;$ IR (film) v: 2909, $2861,2230,1453,1072,745,696 \mathrm{~cm}^{-1}$; HRMS (ESI-TOF) $m / z$ calculated for $\mathrm{C}_{35} \mathrm{H}_{36} \mathrm{~N}_{2} \mathrm{NaO}_{4}$ $\left[\mathrm{M}+\mathrm{Na}^{+}\right], 571.2573$, found 571.2581 .

Copies of ${ }^{1} \mathrm{H}-,{ }^{13} \mathrm{C}-\mathrm{NMR}$, spectra for new compounds: $3 \mathbf{a}, \mathbf{2 - e p i - 3 a}$, and $\mathbf{3 b}$ are available in the Supplementary Materials.

\subsection{Deprotection/Nitrile Reduction-General Procedure for: ADMDP, 2-Epi-ADMDP, and $4 \boldsymbol{b}$}

A mixture of $\mathbf{3 a}$, 2-epi-3a, or $\mathbf{3 b}(0.5 \mathrm{mmol})$, palladium hydroxide $(20 \mathrm{mg})$, and a catalytic amount of acetic acid in methanol $(5 \mathrm{~mL})$ was stirred under a hydrogen atmosphere for $48 \mathrm{~h}$. The reaction mixture was filtered through Celite and the filtrate was concentrated. The residue was purified by chromatography on silica gel to afford the corresponding product ADMDP, 2-epi-ADMDP, and $4 \mathrm{~b}$.

11-Amino-1,2,5-trideoxy-2,5-imino-D-mannitol (ADMDP): colorless oil; isolated yield 73\%; $\mathrm{R}_{f} 0.01$ (DCM/MeOH/EtOH/30\% aq. $\mathrm{NH}_{3}, 5 / 2 / 2 / 1$ ); chromatography ( $25 \%$ aq. $\mathrm{NH}_{4} \mathrm{OH}(37 \%)$ in propanol); $[\alpha]_{\mathrm{D}}+44.1\left(c, 0.35 \mathrm{H}_{2} \mathrm{O}\right)$; agreement with the literature data ${ }^{12 \mathrm{~b}} ;{ }^{1} \mathrm{H} \mathrm{NMR}(500 \mathrm{MHz}$, $2 \% \mathrm{DCl}$ in $\left.\mathrm{D}_{2} \mathrm{O}\right) \delta: 4.08(\mathrm{t}, J 7.0 \mathrm{~Hz}, 1 \mathrm{H}), 4.04(\mathrm{t}, J 7.0 \mathrm{~Hz}, 1 \mathrm{H}), 3.89(\mathrm{dd}, J 12.7,3.9 \mathrm{~Hz}, 1 \mathrm{H}), 3.79$ $(\mathrm{dd}, J 12.7,7.0 \mathrm{~Hz}, 1 \mathrm{H}), 3.73(\mathrm{td}, J 7.2,7.0 \mathrm{~Hz}, 1 \mathrm{H}), 3.61(\mathrm{td}, J 7.0,3.9 \mathrm{~Hz}, 1 \mathrm{H}),, 3.36(\mathrm{~d}, J 7.2 \mathrm{~Hz}, 2 \mathrm{H})$; ${ }^{13} \mathrm{C}$ NMR $\left(125 \mathrm{MHz}, 2 \% \mathrm{DCl}\right.$ in $\left.\mathrm{D}_{2} \mathrm{O}\right)$ 8: 76.4, 73.9, 63.4, 58.4, 58.0, 38.6; IR (film) v: 3209, 2925, 1603, $1503,1406,1123,1065,1034,813 \mathrm{~cm}^{-1}$; HRMS (ESI-TOF) $m / z$ calculated for $\mathrm{C}_{6} \mathrm{H}_{14} \mathrm{~N}_{2} \mathrm{O}_{3}\left[\mathrm{M}+\mathrm{H}^{+}\right]$, 163.1077, found 163.1082 .

2-epi-ADMDP: yellow syrup; isolated yield 69\%; $\mathrm{R}_{f} 0.05$ (DCM/MeOH/EtOH/30\% aq. $\left.\mathrm{NH}_{3}, 5 / 2 / 2 / 1\right)$; chromatography (25\% aq. $\mathrm{NH}_{4} \mathrm{OH}(37 \%)$ in propanol); $[\alpha]_{\mathrm{D}}+12.1\left(c, 0.65 \mathrm{H}_{2} \mathrm{O}\right)$; agreement with the literature data [12b]; ${ }^{1} \mathrm{H}$ NMR $\left(600 \mathrm{MHz}, \mathrm{D}_{2} \mathrm{O}\right) \delta: 3.09(\mathrm{dd}, J 6.3,13.1 \mathrm{~Hz}, 1 \mathrm{H})$, $3.13(\mathrm{q}, J 5.5 \mathrm{~Hz}, 1 \mathrm{H}), 3.24(\mathrm{dd}, J 6.0,13.1 \mathrm{~Hz}, 1 \mathrm{H}), 3.59(\mathrm{q}, J 6.1 \mathrm{~Hz} 1 \mathrm{H}), 3.64(\mathrm{dd}, J 6.5,11.6 \mathrm{~Hz} 1 \mathrm{H})$, $3.74(\mathrm{dd}, J 4.5,11.6 \mathrm{~Hz}, 1 \mathrm{H}), 3.90(\mathrm{dd}, J 3.8,5.3 \mathrm{~Hz}, 1 \mathrm{H}), 4.20(\mathrm{dd}, J 3.8,5.8 \mathrm{~Hz}, 1 \mathrm{H}) ;{ }^{13} \mathrm{C}$ NMR $\left(151 \mathrm{MHz}, \mathrm{D}_{2} \mathrm{O}\right)$ 8: $77.9,76.8,64.0,62.2,55.9,39.2$; HRMS calculated for $\left[\mathrm{C}_{6} \mathrm{H}_{14} \mathrm{~N}_{2} \mathrm{O}_{3}+\mathrm{H}^{+}\right]$ 163.1077, found 163.1079 .

1-Amino-1-deoxy-2,6-dideoxy-2,6-imino-D-glycero-D-ido-heptopyranose (4b): thick oil; isolated yield $66 \%$; Rf 0.06 (DCM/MeOH/EtOH/30\% aq. NH3, 5/2/2/1); chromatography (25\% aq. $\mathrm{NH} 4 \mathrm{OH}(37 \%)$ in propanol); $[\alpha] \mathrm{D}+3.1$ (c, $0.65 \mathrm{H} 2 \mathrm{O})$; agreement with the literature data [38]; 1H NMR (500 MHz, D2O) $\delta: 3.99(\mathrm{dt}, \mathrm{J}$ 7.5, $5.3 \mathrm{~Hz}, 1 \mathrm{H}), 3.94$ (dd, J 9.0, $5.4 \mathrm{~Hz}, 1 \mathrm{H}), 3.91(\mathrm{dd}, \mathrm{J} 13.0,4.8 \mathrm{~Hz}, 1 \mathrm{H}), 3.86(\mathrm{dd}, \mathrm{J} 13.0,3.3 \mathrm{~Hz}, 1 \mathrm{H}), 3.68(\mathrm{t}, \mathrm{J} 9.0 \mathrm{~Hz}, 1 \mathrm{H})$, 3.67 (dd, J 14.0, 7.5 Hz, 1H), 3.58 (dd, J 10.0, 9.0 Hz, 1H), 3.37 (dd, J 14.0 Hz, 5.4 Hz, 1H), 3.26 (ddd, J 10.0, 4.8, 3.3 Hz, 1H); 13C NMR (125 MHz, D2O) 8: 72.8, 69.3, 68.2, 57.9, 57.0, 52.9, 36.7; IR (film) v: 3384, 1618, 1100, 1033, 900, $838 \mathrm{~cm}-1$; HRMS calculated for [C7H16N2O4+H+] 193.1188, found 193.1182 .

4.4. One-Pot Reduction/Enol Silyl Ether Addition to Sugar-Lactams $\mathbf{1 b}$-General Procedure for the Synthesis of $\mathbf{6} \boldsymbol{a}, \boldsymbol{6} \boldsymbol{b}$, and $\mathbf{6} \boldsymbol{c}$

A solution of sugar lactam $\mathbf{1 b}(0.5 \mathrm{mmol})$ in THF $(5 \mathrm{~mL})$ was added dropwise to a suspension of $\mathrm{Cp}_{2} \mathrm{Zr}(\mathrm{H}) \mathrm{Cl}$ (1.6 equiv, $0.8 \mathrm{mmol}, 206 \mathrm{mg}$ ) in THF (5 mL) under an argon atmosphere. The mixture was stirred until the white suspension disappeared (ca. $10 \mathrm{~min}$ ) and a clear solution was formed. Next, the imine solution was cooled to $-25^{\circ} \mathrm{C}$ and $\mathrm{Yb}$ 
$(\mathrm{OTf})_{3}(10 \mathrm{~mol} \%, 0.05 \mathrm{mmol}, 31 \mathrm{mg})$ was added. The mixture was stirred for $10 \mathrm{~min}$ and a solution of enol silyl ether (2.0 equiv $1.0 \mathrm{mmol})$ in THF $(5 \mathrm{~mL})$ was added dropwise. The mixture was gradually warmed to ambient temperature and stirred overnight. Then, the reaction was quenched by the addition of aq. $\mathrm{NaHCO}_{3}$ and stirred for $30 \mathrm{~min}$. After dilution with $\mathrm{Et}_{2} \mathrm{O}(5 \mathrm{~mL})$, the aqueous phase was separated and washed twice with $\mathrm{Et}_{2} \mathrm{O}(2 \times 5 \mathrm{~mL})$. The combined organic layers were dried over anhydrous $\mathrm{MgSO}_{4}$, filtered, and the solvents were removed under reduced pressure to afford the residue, which was chromatographed on silica gel to afford the corresponding product $\mathbf{6 a}, \mathbf{6 b}$, or $\mathbf{6 c}$.

(E)-ethyl-4-((2R,3S,4R,5R,6R)-3,4,5-tris(benzyloxy)-6-(benzyloxymethyl)piperidin-2-yl) but-2-enoate (6a): colorless oil; 88\% (overall, isolated yield for two steps, both isomers); $d r>95: 5$ (determined by ${ }^{1} \mathrm{H}$ NMR of crude reaction mixture); colorless oil; $\mathrm{R}_{f} 0.22$ (1:3 AcOEt/hexanes); chromatography (1:3 AcOEt/hexanes); ${ }^{1} \mathrm{H}$ NMR (600 MHz, $\left.\mathrm{CDCl}_{3}\right)$ 8: 7.36-7.26 (m, 18H), 7.20-7.18 (m, 2H), 6.95 (ddd, J 15.3, 8.5, 6.4 Hz, 1H), 5.90 (d, J 15.6 Hz, 1H), 4.94 (d, J 10.9 $\mathrm{Hz}, 1 \mathrm{H}), 4.85(\mathrm{~d}, J 10.8 \mathrm{~Hz}, 1 \mathrm{H}), 4.80(\mathrm{~d}, J 10.9 \mathrm{~Hz}, 1 \mathrm{H}), 4.69$ (d, J $11.6 \mathrm{~Hz}, 1 \mathrm{H}), 4.64(\mathrm{~d}$, $J 11.6 \mathrm{~Hz}, 1 \mathrm{H}), 4.50(\mathrm{~d}, J 9.2 \mathrm{~Hz}, 1 \mathrm{H}), 4.48(\mathrm{~d}, J 8.1 \mathrm{~Hz}, 1 \mathrm{H}), 4.41(\mathrm{~d}, J 11.9 \mathrm{~Hz}, 1 \mathrm{H}), 4.22-4.13$ (m, 2H), 3.73-3.68 (m, 2H), 3.65-3.62 (m, 1H), 3.54 (dd, J 9.0, 5.8 Hz, 1H), 3.43-3.38 (m, 1H), 3.34-3.28 (m, 1H), 2.98-2.91 (m, 1H), 2.66-2.59 (m, 1H), 2.52-2.44 (m, 1H), $1.27(\mathrm{t}, J 7.1 \mathrm{~Hz}, 3 \mathrm{H})$; ${ }^{13} \mathrm{C} \mathrm{NMR}\left(151 \mathrm{MHz}, \mathrm{CDCl}_{3}\right) \delta: 166.3,146.5,138.8,138.3,138.0,128.43,128.40,128.36,128.02$, $127.92,127.81,127.76,127.73,127.70,127.63,127.56,123.6,83.1,81.8,80.2,75.6,75.2,73.2,72.8$, 69.9, 60.2, 53.4, 52.8, 29.7, 28.8, 14.2; IR (film) v: 2922, 2860, 1717, 1454, 1096, 1068, 736, $697 \mathrm{~cm}^{-1}$; HRMS (ESI-TOF) $\mathrm{m} / \mathrm{z}$ calculated for $\mathrm{C}_{40} \mathrm{H}_{48} \mathrm{NO}_{6}\left[\mathrm{M}+\mathrm{H}^{+}\right] 636.3325$, found 636.3329.

(S)-5-((2R,3S,4S,5R,6R)-3,4,5-tris(benzyloxy)-6-(benzyloxymethyl)piperidin-2-yl) furan-2(5H)-one (6b): (major isomer), colorless oil; 68\% (overall, isolated yield for two steps, both isomers); $d r$ 75:25 (determined by ${ }^{1} \mathrm{H}$ NMR of crude reaction mixture); colorless oil; $\quad R_{f} 0.43$ (1:1 AcOEt/hexanes); chromatography (1:3 AcOEt/hexanes than 1:1 AcOEt/hexanes); ${ }^{1} \mathrm{H}$ NMR $\left(600 \mathrm{MHz}, \mathrm{CDCl}_{3}\right)$ 8: 7.68-7.66 (m, 1H), 7.36-7.24 (m, 18H), 7.20-7.17 (m, 2H), $6.06(\mathrm{dd}, J 5.7,1.8 \mathrm{~Hz}, 1 \mathrm{H}), 5.48(\mathrm{~d}, J 7.7 \mathrm{~Hz}, 1 \mathrm{H}), 4.94(\mathrm{~d}, J 10.9 \mathrm{~Hz}$, $1 \mathrm{H}), 4.86(\mathrm{~d}, J 7.3 \mathrm{~Hz}, 1 \mathrm{H}), 4.84(\mathrm{~d}, J 7.4 \mathrm{~Hz}, 1 \mathrm{H}), 4.76(\mathrm{~d}, J 11.5 \mathrm{~Hz}, 1 \mathrm{H}), 4.60(\mathrm{~d}, J 11.5 \mathrm{~Hz}$, $1 \mathrm{H}), 4.53-4.49(\mathrm{~m}, 2 \mathrm{H}), 4.43(\mathrm{~d}, J 12.0 \mathrm{~Hz}, 1 \mathrm{H}), 3.87(\mathrm{~d}, J 8.7 \mathrm{~Hz}, 1 \mathrm{H}), 3.84(\mathrm{~d}, J 5.6 \mathrm{~Hz}, 1 \mathrm{H})$, $3.60(\mathrm{dd}, J$ 9.5, $2.5 \mathrm{~Hz}, 1 \mathrm{H}), 3.48(\mathrm{dd}, J 9.5,6.0 \mathrm{~Hz}, 1 \mathrm{H}), 3.43(\mathrm{t}, J 9.1 \mathrm{~Hz}, 1 \mathrm{H}), 3.23-3.20(\mathrm{~m}$, 1H), 3.19-3.15 (m, 1H); $\left.{ }^{13} \mathrm{C} \mathrm{NMR} \mathrm{(151} \mathrm{MHz,} \mathrm{CDCl}_{3}\right) \delta: 172.6,156.6,138.4,138.1,137.8,137.6$, $128.5,128.42,128.40,128.3,128.0,127.95,127.94,127.88,127.86,127.72,127.70,127.68,120.8$, 83.3, 81.2, 80.2, 79.8, 75.6, 75.0, 73.8, 73.1, 69.8, 58.2, 54.3; IR (film) v: 3030, 2865, 1757, 1454, $1091,738,698 \mathrm{~cm}^{-1}$; HRMS (ESI-TOF) $\mathrm{m} / \mathrm{z}$ calculated for $\mathrm{C}_{38} \mathrm{H}_{40} \mathrm{NO}_{6}\left[\mathrm{M}+\mathrm{H}^{+}\right] 602.2856$, found 606.2856; (2-epi-6b) (minor isomer) selected signals: ${ }^{1} \mathrm{H}$ NMR $\left(600 \mathrm{MHz}, \mathrm{CDCl}_{3}\right) \delta$ : $7.70(\mathrm{~d}, J 5.4 \mathrm{~Hz}, 1 \mathrm{H}), 6.10(\mathrm{dd}, J 5.7,1.9 \mathrm{~Hz}, 1 \mathrm{H}), 5.30(\mathrm{~d}, J 6.5 \mathrm{~Hz}, 1 \mathrm{H}) ;{ }^{13} \mathrm{C} \mathrm{NMR}(151 \mathrm{MHz}$, $\left.\mathrm{CDCl}_{3}\right) \delta: 173.0,156.2,138.1,138.0,137.9,137.7,121.4,55.2,54.8$.

1-(4-Fuorophenyl)-2-((2R,3S,4R,5R,6R)-3,4,5-tris(benzyloxy)-6-(benzyloxymethyl)

piperidin-2-yl)ethanone (6c): colorless oil; 43\% (overall, isolated yield for two steps, both isomers); $d r>95: 5$ (determined by ${ }^{1} \mathrm{H}$ NMR of crude reaction mixture); $\mathrm{R}_{f} 0.32$ (1:3 AcOEt/hexanes); chromatography (1:4 AcOEt/hexanes); ${ }^{1} \mathrm{H}$ NMR $\left(600 \mathrm{MHz}, \mathrm{CDCl}_{3}\right)$

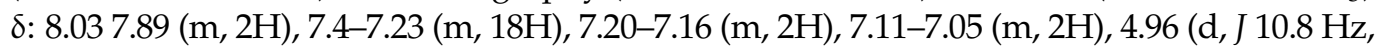
$1 \mathrm{H}), 4.85(\mathrm{~d}, J 10.5 \mathrm{~Hz}, 1 \mathrm{H}), 4.82(\mathrm{~d}, J 10.9 \mathrm{~Hz}, 1 \mathrm{H}), 4.66(\mathrm{~d}, J 11.5 \mathrm{~Hz}, 1 \mathrm{H}), 4.63$ (d, J $3.8 \mathrm{~Hz}$, $1 \mathrm{H}), 4.50-4.41(\mathrm{~m}, 3 \mathrm{H}), 4.10-4.05(\mathrm{~m}, 1 \mathrm{H}), 3.80-3.76(\mathrm{~m}, 1 \mathrm{H}), 3.74-3.69(\mathrm{~m}, 1 \mathrm{H}), 3.61$ (dd, J 9.1, $2.3 \mathrm{~Hz}, 1 \mathrm{H}), 3.53-3.49(\mathrm{~m}, 1 \mathrm{H}), 3.45-3.39(\mathrm{~m}, 1 \mathrm{H}), 3.33(\mathrm{dd}, J 16.9,3.5 \mathrm{~Hz}, 1 \mathrm{H}), 3.18$ (dd, J 16.9, $9.5 \mathrm{~Hz}, 1 \mathrm{H}), 3.00-2.94(\mathrm{~m}, 1 \mathrm{H}) ;{ }^{13} \mathrm{C} \mathrm{NMR}\left(151 \mathrm{MHz}, \mathrm{CDCl}_{3}\right) \delta: 197.6,165.75(\mathrm{~d}, J=254.8 \mathrm{~Hz})$, 138.7, 138.2, 138.1, 137.9, 130.75 (d, $J=9.3 \mathrm{~Hz}), 128.41,128.37,128.34,128.0,127.9,127.84,127.77$, $127.75,127.71,127.65,127.57,115.64(\mathrm{~d}, J=21.9 \mathrm{~Hz}), 75.6,75.3,73.2,72.9,53.5,50.6,34.5$; IR (film) v: 3031, 2918, 2862, 1681, 1597, 1453, 1230, 1095, 1068, 737, $698 \mathrm{~cm}^{-1}$; HRMS (ESI-TOF) $\mathrm{m} / z$ calculated for $\mathrm{C}_{42} \mathrm{H}_{43} \mathrm{NO}_{5} \mathrm{~F}\left[\mathrm{M}+\mathrm{H}^{+}\right] 660.3125$, found 660.3124 .

Copies of ${ }^{1} \mathrm{H}-,{ }^{13} \mathrm{C}-\mathrm{NMR}$, spectra for new compounds: $\mathbf{6 a}, \mathbf{6 b}$, and $\mathbf{6 c}$ are available in the Supplementary Materials. 
Supplementary Materials: The followings are available online, Copies of ${ }^{1} \mathrm{H}-,{ }^{13} \mathrm{C}-\mathrm{NMR}$, spectra for new compounds: 3a, 2-epi-3a, 3b, 6a, 6b, 6c.

Author Contributions: Conceptualization, P.S., B.G. and B.F.; methodology, P.S. and B.G.; software, P.S.; validation, P.S. and B.G.; formal analysis, P.S. and B.G.; investigation, P.S. and B.G.; resources, P.S., B.G. and B.F.; data curation, P.S., B.G. and B.F.; writing-original draft preparation, P.S.; writingreview and editing, B.F.; visualization, P.S. All authors have read and agreed to the published version of the manuscript.

Funding: P. Szcześniak is grateful to Polish National Science Center for financial support of the research (Sonata 15 2019/35/D/ST4/00028). B. Furman is grateful to Polish National Science Center for financial support of the research (OPUS 2019/35/B/ST4/02794).

Data Availability Statement: The data is not available from the authors.

Conflicts of Interest: The authors declare no conflict of interest.

Sample Availability: Samples of the compounds are not available from the authors.

\section{References}

1. Stutz, A.E. Iminosugars as Glycosidase Inhibitors: Nojirimycin and beyond; Wiley-VCH: Weinheim, Germany, 1999.

2. Wei, Z. C-Glycosides and Aza-C-Glycosides as Potential Glycosidase and Glycosyltransferase Inhibitors. Curr. Top. Med. Chem. 2005, 5, 1363-1391.

3. Watson, A.A.; Fleet, G.W.J.; Asano, N.; Molyneux, R.J.; Nash, R.J. Polyhydroxylated alkaloids-Natural occurrence and therapeutic applications. Phytochemistry 2001, 56, 265-295. [CrossRef]

4. Winchester, B.; Fleet, G.W.J. Amino-sugar glycosidase inhibitors: Versatile tools for glycobiologists. Glycobiology 1992, 2, 199-210. [CrossRef]

5. Zamoner, L.O.B.; Aragão-Leoneti, V.; Carvalho, I. Iminosugars: Effects of Stereochemistry, Ring Size, and N-Substituents on Glucosidase Activities. Pharmaceuticals 2019, 12, 108. [CrossRef] [PubMed]

6. Nash, R.J.; Kato, A.; Yu, C.-Y.; Fleet, G.W. Iminosugars as therapeutic agents: Recent advances and promising trends. Future Med. Chem. 2011, 3, 1513-1521. [CrossRef]

7. Horne, G.; Wilson, F.X. Therapeutic Applications of Iminosugars: Current Perspectives and Future Opportunities. Prog. Med. Chem. 2011, 50, 135-176.

8. Lillelund, V.H.; Jensen, H.H.; Liang, X.; Bols, M. Recent Developments of Transition-State Analogue Glycosidase Inhibitors of Non-Natural Product Origin. Chem. Rev. 2002, 102, 515-554. [CrossRef]

9. Greimel, P.; Spreitz, J.; Stütz, A.E.; Wrodnigg, T.M. Iminosugars and relatives as antiviral and potential anti-infective agents. Curr. Med. Chem. 2003, 3, 513-523. [CrossRef]

10. Legler, G.; Korth, A.; Berger, A.; Ekhart, C.; Gradnig, G.; Stütz, A.E. 2,5-dideoxy-2,5-imino-d-mannitol and -d-glucitol. Twostep bio-organic syntheses from 5-azido-5-deoxy-d-glucofuranose and -l-idofuranose; evaluation as glucosidase inhibitors and application in affinity purification and characterisation of invertase from yeast. Carbohydr. Res. 1993, 250, 67-77.

11. Aoyagi, S.; Fujimaki, S.; Kibayashi, C. Total synthesis of (+)- $\alpha$-homonojirimycin. J. Chem. Soc. Chem. Commun. 1990, 20, 1457-1459. [CrossRef]

12. Takaoka, Y.; Kajimoto, T.; Wong, C.H. Inhibition of N-acetylglucosaminyl transfer enzymes: Chemical-enzymic synthesis of new five-membered acetamido azasugars. J. Org. Chem. 1993, 58, 4809-4812. [CrossRef]

13. Liu, J.; Numa, M.M.D.; Liu, H.; Huang, S.-J.; Sears, P.; Shikhman, A.R.; Wong, C.-H. Synthesis and High-Throughput Screening of N-Acetyl- $\beta$-hexosaminidase Inhibitor Libraries Targeting Osteoarthritis. J. Org. Chem. 2004, 69, 6273-6283. [CrossRef]

14. Takebayashi, M.; Hiranuma, S.; Kanie, Y.; Kajimoto, T.; Kanie, O.; Wong, C.-H. A Versatile Synthetic Strategy for the Preparation and Discovery of New Iminocyclitols as Inhibitors of Glycosidases. J. Org. Chem. 1999, 64, 5280-5291. [CrossRef]

15. Saotome, C.; Wong, C.-H.; Kanie, O. Combinatorial library of five-membered iminocyclitol and the inhibitory activities against glyco-enzymes. Chem. Biol. 2001, 8, 1061-1070. [CrossRef]

16. Liang, P.-H.; Cheng, W.-C.; Lee, Y.-L.; Yu, H.-P.; Wu, Y.-T.; Lin, Y.-L.; Wong, C.-H. Novel Five-Membered Iminocyclitol Derivatives as Selective and Potent Glycosidase Inhibitors: New Structures for Antivirals and Osteoarthritis. ChemBioChem 2006, 7, 165-173. [CrossRef] [PubMed]

17. Shih, H.-W.; Chen, K.-T.; Chen, S.-K.; Huang, C.-Y.; Cheng, T.-J.R.; Ma, C.; Wong, C.-H.; Cheng, W.-C. Combinatorial approach toward synthesis of small molecule libraries as bacterial transglycosylase inhibitors. Org. Biomol. Chem. 2010, 8, $2586-2593$. [CrossRef] [PubMed]

18. Kang, S.H.; Ryu, D.H. Intramolecular cyclization of C2 symmetric and meso-iodo amino alcohols: A synthetic approach to azasugars. Tetrahedron Lett. 1997, 38, 607-610. [CrossRef]

19. Popowycz, F.; Gerber-Lemaire, S.; Schütz, C.; Vogel, P. Syntheses and Glycosidase Inhibitory Activities of 2-(Aminomethyl)-5(hydroxymethyl)pyrrolidine-3,4-diol Derivatives. Helv. Chim. Acta 2004, 87, 800-810. [CrossRef] 
20. McCort, I.; Fort, S.; Duréault, A.; Depezay, J.-C. Synthesis and evaluation as glycosidase inhibitors of 2,5-imino-d-glucitol and 1,5-imino-d-mannitol related derivatives. Bioorg. Med. Chem. 2000, 8, 135-143. [CrossRef]

21. Merino, P.; Delso, I.; Tejero, T.; Cardona, F.; Marradi, M.; Faggi, E.; Parmeggiani, C.; Goti, A. Nucleophilic Additions to Cyclic Nitrones en Route to Iminocyclitols-Total Syntheses of DMDP, 6-deoxy-DMDP, DAB-1, CYB-3, Nectrisine, and Radicamine B. Eur. J. Org. Chem. 2008, 2008, 2929-2947. [CrossRef]

22. Tsou, E.-L.; Yeh, Y.-T.; Liang, P.-H.; Cheng, W.-C. A convenient approach toward the synthesis of enantiopure isomers of DMDP and ADMDP. Tetrahedron 2009, 65, 93-100. [CrossRef]

23. Win-Mason, A.L.; Jongkees, S.A.K.; Withers, S.G.; Tyler, P.C.; Timmer, M.S.M.; Stocker, B.L. Stereoselective Total Synthesis of Aminoiminohexitols via Carbamate Annulation. Org. Chem. 2011, 76, 9611-9621. [CrossRef]

24. Ganesan, M.; Madhukarrao, R.V.; Ramesh, N.G. Design and synthesis of new amino-modified iminocyclitols: Selective inhibitors of $\alpha$-galactosidase. Org. Biomol. Chem. 2010, 8, 1527-1530. [CrossRef] [PubMed]

25. Szcześniak, P.; Stecko, S.; Staszewska-Krajewska, O.; Furman, B. Sugar-derived cyclic imines: One-pot synthesis and direct functionalization. Tetrahedron 2014, 70, 1880-1888. [CrossRef]

26. Szcześniak, P.; Stecko, S.; Maziarz, E.; Staszewska-Krajewska, O.; Furman, B. Synthesis of Polyhydroxylated Quinolizidine and Indolizidine Scaffolds from Sugar-Derived Lactams via a One-Pot Reduction/Mannich/Michael Sequence. Org. Chem. 2014, 79, 10487-10503. [CrossRef]

27. Szcześniak, P.; Maziarz, E.; Stecko, S.; Furman, B. Synthesis of Polyhydroxylated Piperidine and Pyrrolidine Peptidomimetics via One-Pot Sequential Lactam Reduction/Joullié-Ugi Reaction. Org. Chem. 2015, 80, 3621-3633. [CrossRef]

28. Hoos, R.; Naughton, A.B.; Vasella, A. A Convenient Procedure for he Synthesis o 2,3,4,6-Tetra-O-benzyl-D-gluconolactam and D-Nojirilactam. Helv. Chim. Acta 1993, 76, 1802-1807. [CrossRef]

29. Larsen, C.H.; Ridgway, B.H.; Shaw, J.T.; Woerpel, K.A. A Stereoelectronic Model To Explain the Highly Stereoselective Reactions of Nucleophiles with Five-Membered-Ring Oxocarbenium Ions. Am. Chem. Soc. 1999, 121, 12208-12209. [CrossRef]

30. Ayala, L.; Lucero, C.G.; Romero, J.A.C.; Tabacco, S.A.; Woerpel, K.A. Stereochemistry of Nucleophilic Substitution Reactions Depending upon Substituent: Evidence for Electrostatic Stabilization of Pseudoaxial Conformers of Oxocarbenium Ions by Heteroatom Substituents. Am. Chem. Soc. 2003, 125, 15521-15528. [CrossRef] [PubMed]

31. Larsen, C.H.; Ridgway, B.H.; Shaw, J.T.; Smith, D.M.; Woerpel, K.A. Stereoselective C-Glycosylation Reactions of Ribose Derivatives: Electronic Effects of Five-Membered Ring Oxocarbenium Ions. Am. Chem. Soc. 2005, 127, 10879-10884. [CrossRef]

32. Lucero, C.G.; Woerpel, K.A. Stereoselective C-Glycosylation Reactions of Pyranoses: The Conformational Preference and Reactions of the Mannosyl Cation. Org. Chem. 2006, 71, 2641-2647. [CrossRef] [PubMed]

33. Yang, M.T.; Woerpel, K.A. The Effect of Electrostatic Interactions on Conformational Equilibria of Multiply Substituted Tetrahydropyran Oxocarbenium Ions. Org. Chem. 2009, 74, 545-553. [CrossRef] [PubMed]

34. Tran, V.T.; Woerpel, K.A. Nucleophilic Addition to Silyl-Protected Five-Membered Ring Oxocarbenium Ions Governed by Stereoelectronic Effects. Org. Chem. 2013, 78, 6609-6621. [CrossRef] [PubMed]

35. Shenoy, S.R.; Smith, D.M.; Woerpel, K.A. Nucleophilic Additions of Trimethylsilyl Cyanide to Cyclic Oxocarbenium Ions: Evidence for the Loss of Stereoselectivity at the Limits of Diffusion Control. Am. Chem. Soc. 2006, 128, 8671-8677. [CrossRef]

36. Rychnovsky, S.D.; Dahanukar, V.H. Carbon-Carbon Bond Formation from Small- and Medium-Ring Lactol Acetates via Radical and Oxonium Ion Intermediates. Synthesis of ( \pm )-Laurenan. Org. Chem. 1996, 61, 7648-7649. [CrossRef] [PubMed]

37. Revuelta, J.; Cicchi, S.; Goti, A.; Brandi, A. Enantiopure Cyclic Nitrones: A Useful Class of Building Blocks for Asymmetric Syntheses. Synthesis 2007, 2007, 485-504. [CrossRef]

38. Wong, C.-H.; Provencher, L.; Porco, J.A.; Jung, S.-H.; Wang, Y.-F.; Chen, L.; Wang, R.; Steensma, D.H. Synthesis and Evaluation of Homoaza Sugars as Glycosidase Inhibitors. J. Org. Chem. 1995, 60, 1492-1501. [CrossRef] 